\title{
OBSERVACIONES DE FRUTOS Y SEMILLAS EN TRES ESPECIES DE ODONTITES LUDWIG (SCROPHULARIACEAE)
}

\author{
Rocio JUAN, Julio PASTOR e Inmaculada FERNÁNDEZ
}

\begin{abstract}
RESUMEN. Observaciones de frutos y semillas en tres especies de Odontites Ludwig (Srophulariaceae). Se ha realizado un estudio morfológico y anatómico en frutos y semillas de tres especies de Odontites Ludwig ( $O$. tenuifolia, $O$. longiflora y $O$. foliosa). Los resultados muestran que estos taxones pueden ser diferenciados por los frutos y las semillas. Sin embargo, los caricteres utilizados no apoyan la separación de estas especies en géneros diferentes (Odontitella y Macrosyringion).
\end{abstract}

Palabras claves. Fruto, Semilla, Anatomía, Morfología, Odontites, Odontitella, Macrosyringion, Scrophulariaceale

ABSTRACT. Fruits and seeds observations in three species of Odontites Ludwig (Scrophulariaceae). A morphological and anatomical study of fruits and seeds of three species of Odontites Ludwig (O. tenuifolia, $O$. longiflora and $O$. foliosa) has been made. The results show that these taxa can be differentiated on the basis of fruits and seeds features. However, the characters used do not support separation these species in different genera (Odontitella and Macrosyringion).

Key words. Fruit, Seed, Anatomy, Morphology, Odontites, Odontitella, Macrosyringion, Scrophulariaceae.

\section{INTRODUCCIÓN}

El género Odontites Ludwig reune alrededor de 25 - 30 especies confinadas en su mayoría al oeste de la región mediterránea (Bolliger \& Wick, 1990). Tiene una estrecha relación con Euphrasia y Bartsia, Linneo (1753) lo incluyó en Euphrasia y Bentham (1846) lo trató como una sección de Bartsia. En la Península Ibérica, Webb \& Camarasa (1972) reconocen nueve especies, tres de las cuales han sido objeto de este estudio.

Son hierbas anuales, hemiparásitas o matas, que crecen generalmente en lugares secos. Tienen hojas opuestas, estrechas y sentadas. Flores en racimo unilateral bracteado. Cáliz tubuloso con 4 lóbulos. Corola zigomorfa con tubo cilíndrico, labio superior entero o apenas bilobado y labio inferior trilobado. Androceo con 4 estambres, didínamo. Estigma capitado. Cápsula loculicida y semillas ápteras, estriadas longitudinalmente.

El enorme polimorfismo de este género puesto de manifiesto por Rothmaler (1943), ha motivado una revisión reciente de Bolliger (1985). Apenas existen trabajos sobre este género, destacando el estudio polínico realizado por Bolliger \& Wick (1990) con el fin de clarificar la sistemática del género y sus relaciones con otros afines. Después de estos 
estudios morfológicos y sistemáticos, el género Odontites s.l. queda fragmentado en varios géneros formados por una o dos especies, a excepción de Odontites s.s. que presenta unas 20 especies. Según estos autores, Odontites s.s. estaría constituido por aquellos taxones con gálea emarginada, más larga que el labio, estigma entero, polen con exina microrreticulada y número básico $x=10,12$. Por otro lado, Macrosyringion incluiría las especies que tienen gálea entera, más corta que el labio, estigma bilobado, polen con exina retipilada y $\mathrm{x}=12$. Por último, Odontitella englobaría las especies con gálea entera, aunque de igual longitud que el labio, estigma bilobado, polen con exina retipilada y $\mathrm{x}=13$.

Aunque en este estudio se ha seguido el tratamiento taxonómico de Valdés (1987), según Bolliger (1985) y Bolliger \& Molau (1992) O. tenuifolia aparece como Odontitella tenuifolia, $O$. longiflora como Macrosyringion longiflorum y sólo $O$. foliosa permanece en Odontites s.s. Por este motivo se han elegido estos tres taxones para el presente estudio de frutos y semillas, cuya finalidad ha sido comprobar si los caracteres examinados apoyan la separación de estas especies en géneros distintos.

\section{MATERIAL Y MÉTODOS}

Se han estudiado 10 poblaciones pertenecientes a tres especies de Odontites. Los testigos se encuentran depositados en el Herbario del Departamento de Biología Vegetal y Ecología de la Facultad de Biología de Sevilla (SEV).

El estudio morfológico se llevó a cabo con cápsulas y semillas rehidratadas que posteriormente se sometieron a punto crítico usando dióxido de carbono. Estos frutos y semillas junto a semillas secas se montaron en portas mediante adhesivo de doble cara. A continuación se metalizaron con oro-paladio y se examinaron con un microscopio electrónico de barrido (MEB). Los datos de longitud y anchura están basados en un muestreo de 35 90 cápsulas o semillas por taxón. El número de semillas por cápsulas únicamente se ha podido contabilizar en $O$. longiflora ya que ha sido la única especie de la que se ha dispuesto de cápsulas sin abrir. Para determinar la variabilidad intra e interpoblacional se aclararon y reblandecieron en ácido láctico, varias cápsulas de cada población para comparar las epidermis. Éstas se montaron en portas y se observaron al microscopio óptico (MO), donde se efectuaron las medidas de los pelos. Con ayuda de una cámara clara se realizaron los dibujos de las cápsulas completas así como de los detalles de las epidermis y pelos.

En el estudio anatómico se han utilizado cápsulas y semillas hervidas en agua durante 3-5 minutos. Seguidamente este material se deshidrató usando las series de alcohol butílico terciario (Johansen, 1940). A continuación se incluyeron en parafina y se cortaron a 8-10 $\mu \mathrm{m}$. Una vez montadas estas secciones se tiñeron con safranina alcohólica al $1 \%$ y fast green alcohólico al $0.1 \%$. Estas preparaciones se montaron de modo permanente para su posterior estudio al MO.

Para la terminología se ha seguido fundamentalmente a Font Quer (1977) y Stearn (1992).

\section{Material estudiado.}

O. tenuifolia: BURGOS. Bozoo, 31.8.1983, UribeEchebarría \& Urrutia (SEV 118128). CÁDIZ. Los Barrios, Sierra de La Palma, 28.9.1982, Allen (SEV 88631). SORIA. Borobia, 2.8.1974, Segura Zubizarreta (SEV 27010).

O. Iongiflora: GRANADA. Gorafe, 25.9.1971, Fernández Casas (SEV 112541). Güejar Sierra, Púlpito de Canales, 19.10.1979, Ladero, López \& Molero Mesa (SEV 55577). Sierra Nevada, 

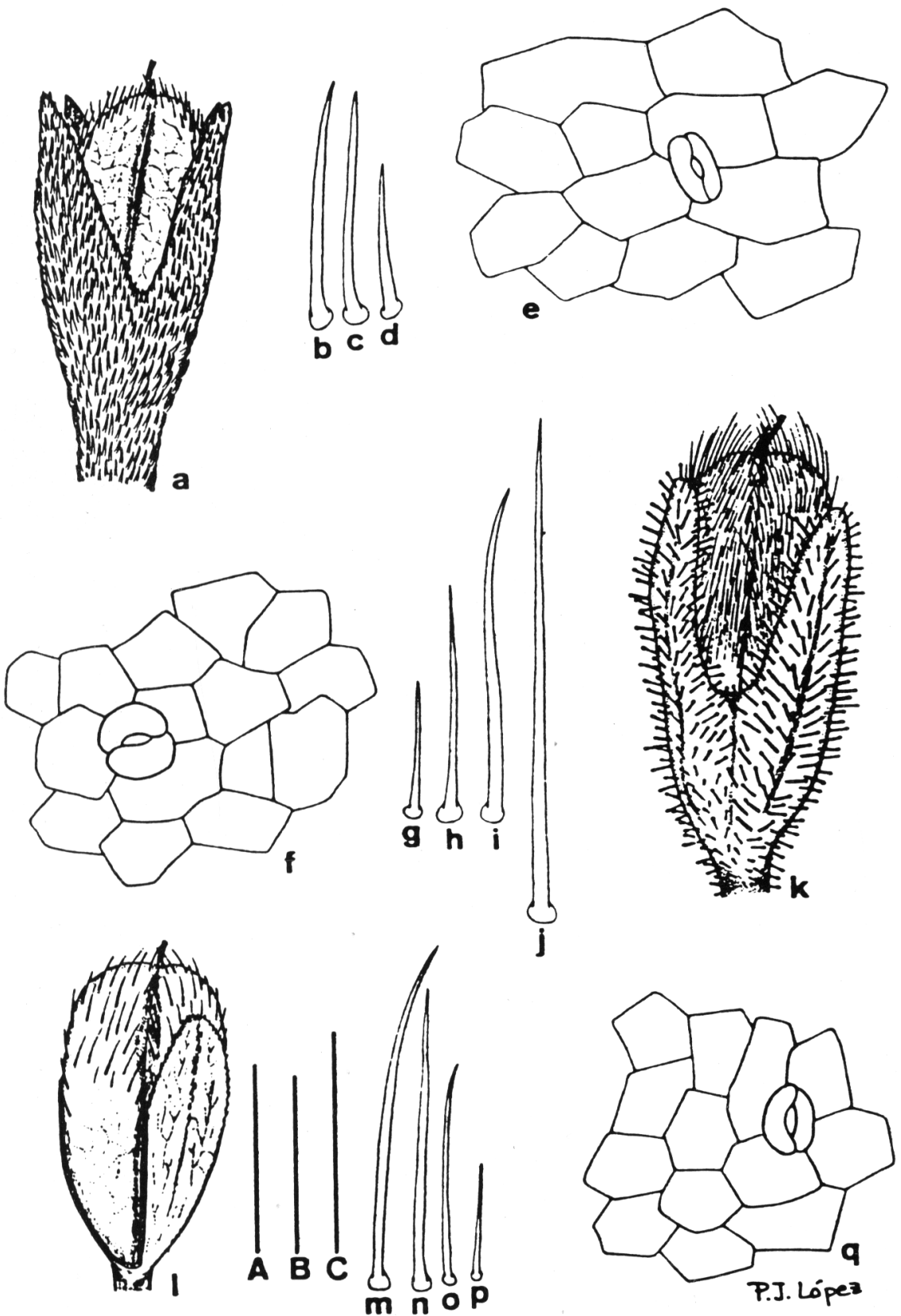

Figura 1. Odontites tenuifolia: a-e; $O$. longiflora: $\mathrm{f}-\mathrm{k} ;$ O. foliosa: 1-q. a, k, 1: aspecto general de la cápsula; b-d, g-j, m-p: tricomas eglandulares; e, f, q: detalle de la superficie. Escalas: $\mathrm{A}=3 \mathrm{~mm}(\mathrm{a}, \mathrm{k}, \mathrm{l}) ; \mathrm{B}=250$ $\mu \mathrm{m}(\mathrm{b}-\mathrm{d}, \mathrm{g}-\mathrm{j}, \mathrm{m}-\mathrm{p}) ; \mathrm{C}=100 \mu \mathrm{m}(\mathrm{e}, \mathrm{f}, \mathrm{l}, \mathrm{q})$. Odontites tenuifolia: $a-e ; O$. longiflora: $f-k ; O$. foliosa: $l-q . a$, $k$, l: drawings of capsule; $b-d, g-j, m-p$ : eglandular hairs; $e, f, q$ : detail of surface. Scale bars: $A=3 \mathrm{~mm}$ $(a, k, l) ; B=250 \mu m(b-d, g-j, m-p) ; C=100 \mu m(e, f, l, q)$. 
desvío al Dornajo, 20.10.1992, Fernández \& Juan (SEV 135374). JAÉN. Sierra de Cazorla, La Iruela, 22.9.1973, Heywood \& Moore (SEV 28350).

O. foliosa: CÁDIZ. Algeciras, Las Habas, 1.11.1973, Allen (SEV 97133). Entre Manilva y San Martín del Tesorillo, 23.11.1979, Allen (SEV 88189). MÁLAGA. Manilva, cerro del Castillo, 7.11.1976, Smythies (SEV 26039).

\section{RESULTADOS}

Las tres especies estudiadas muestran cápsulas coriáceas, solitarias y dispuestas en racimos densos. Tienen simetría bilateral y están formadas por dos lóbulos subiguales con margen aquillado. La superficie presenta células poligonales, convexas, a veces con márgenes engrosados. La cutícula es rugulosa y con frecuencia se observan estomas. El indumento está constituido por pelos unicelulares eglandulares lisos, con la base ensanchada sobre una célula epidérmica de mayor tamaño. El epicarpo y el mesocarpo muestran células de paredes delgadas, mientras que el endocarpo las tiene lignificadas. La dehiscencia es loculicida sin superar la mitad del fruto.

Las semillas tienen inserción apical y testa crestada. Tanto la epidermis como la hipodermis presentan células con paredes reforzadas. En ninguna especie examinada se ha observado rafe.

A continuación se describen las especies estudiadas:

Odontites tenuifolia (Pers.) G. Don

Cápsulas de $4.8-7.0 \times 2.0-2.9 \mathrm{~mm}$. De contorno estrechamente obovado u oblongo, comprimidas, con ápice truncado algo emarginado (fig. 1 a). Indumento pubescente, adpreso, localizado en el ápice y en los márgenes de la mitad superior, con pelos de 100 - $400 \mu \mathrm{m}$ (Figs. 1 b-e; 2 a,d). Pericarpo de $60-120 \mu \mathrm{m}$. Epicarpo con células irregulares de paredes delgadas. Mesocarpo con 2 - 3 capas de células irregulares, colapsadas con frecuencia. Endocarpo de $20-30 \mu \mathrm{m}$, con 2 capas de células cuadradas, pequeñas y lignificadas (fig. 2 b).

Semillas de $1.1-1.6$ × $0.5-0.9$ mm, oblongoideas o elipsoideas. Color pardo claro. Testa crestada, constituida por células rectangulares, con paredes radiales reticuladas poco o medianamente profundas, de grosor irregular y pared tangencial externa membranosa colapsada sobre la interna que es reticulada. Entre cada cresta se disponen 2 filas de células, a veces 1 , dando un aspecto más o menos reticulado. Epidermis con células irregulares de paredes con refuerzos de lignina excepto la tangencial externa. Hipodermis con 1 - 2 capas de células irregulares de paredes reforzadas. Endotelio con células colapsadas (fig. 2 c,e,f).

\section{Odontites longiflora (Vahl) Webb}

Cápsulas de $6.3-9.2$ × $2.1-3.2 \mathrm{~mm}$. De contorno estrechamente obovado u oblongo, comprimidas, con ápice truncado algo emarginado (fig. $1 \mathrm{k}$ ). Indumento densamente pubescente, adpreso, localizado en la mitad superior, con pelos de 130 - $500 \mu \mathrm{m}$ (Figs. 1 fj; 2 g). Pericarpo de 80 - $160 \mu \mathrm{m}$. Epicarpo con células redondeadas de paredes delgadas. Mesocarpo con 2 - 5 capas de células redondeadas de paredes delgadas. Endocarpo

Figura 2. Odontites tenuifolia: a-f; $O$. longiflora: g-l; $O$. foliosa: m-q. a: superficie de la cápsula; d, g, m: tricoma eglandular; $\mathrm{b}, \mathrm{h}, \mathrm{n}$ : detalle del pericarpo; e, $\mathrm{k}$, p: detalle de la testa; c, i, o: aspecto general de la semilla; f, l, q: superficie de la testa; $\mathrm{j}$ : superficie de la testa en semilla tratada con punto crítico. Escalas: $50 \mu \mathrm{m}(\mathrm{a}, \mathrm{e}, \mathrm{f}, \mathrm{k}, \mathrm{m}, \mathrm{p}, \mathrm{q}) ; 100 \mu \mathrm{m}(\mathrm{b}, \mathrm{h}, \mathrm{j}, \mathrm{l}, \mathrm{n}) ; 200 \mu \mathrm{m}(\mathrm{d}, \mathrm{g}) ; 500 \mu \mathrm{m}(\mathrm{c}, \mathrm{i}, \mathrm{o})$. Odontites tenuifolia: a$f$; O. longiflora: $g$-l; $O$. foliosa: $m-q$. a: surface of capsule; $d, g, m$ : eglandular hair; $b, h, n$ : detail of pericarp; $e, k, p$ : detail of seed coat; $c, i, o$ : whole seed; $f, l, q$ : surface of seed-coat; $j$ : surface of seed-coat treated with critical point. Scale bars: $50 \mu m(a, e, f, k, m, p, q) ; 100 \mu m(b, h, j, l, n) ; 200 \mu m(d, g) ; 500$ $\mu m(c, i, o)$. 

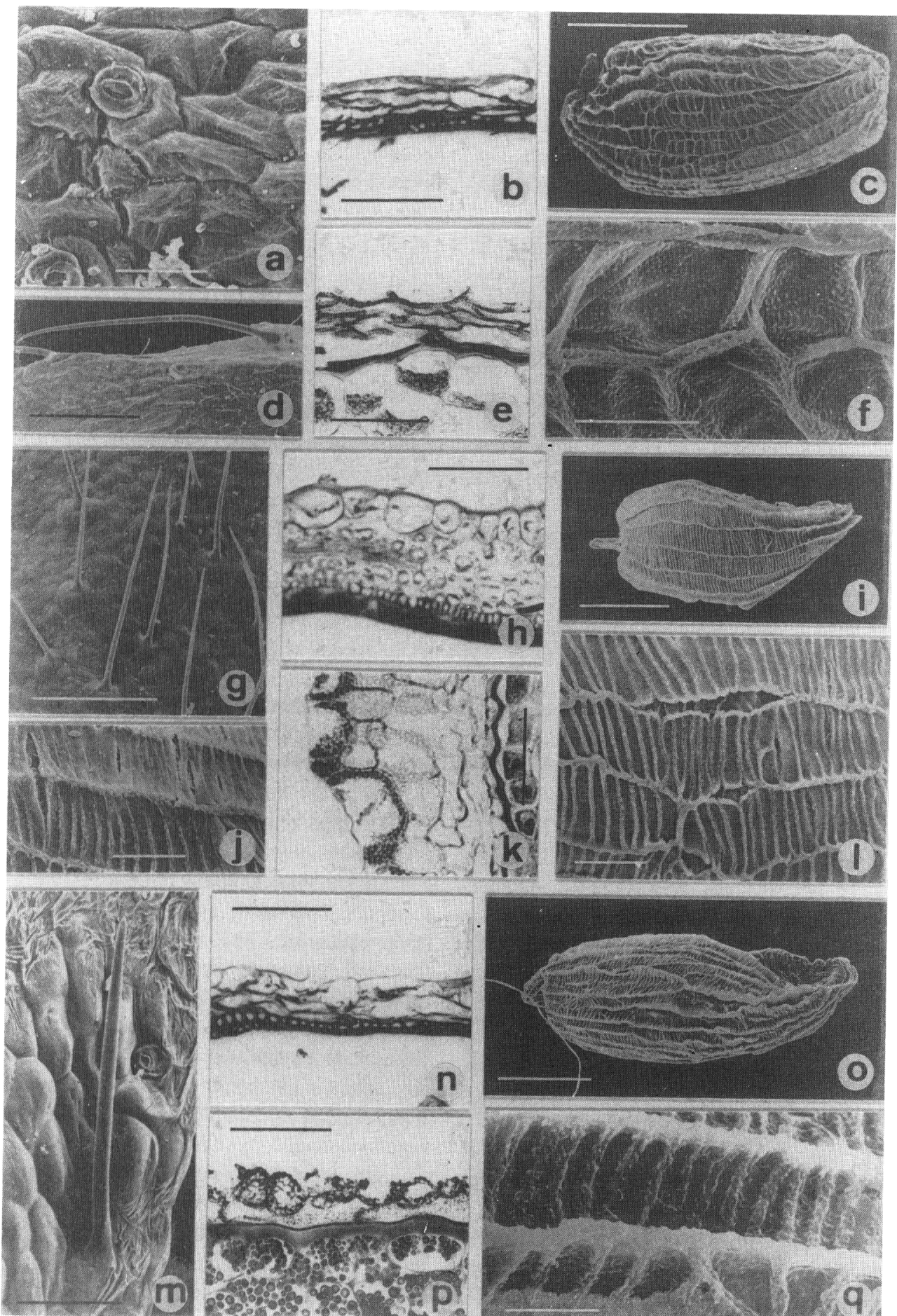

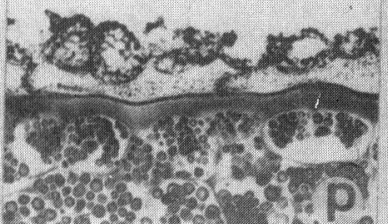

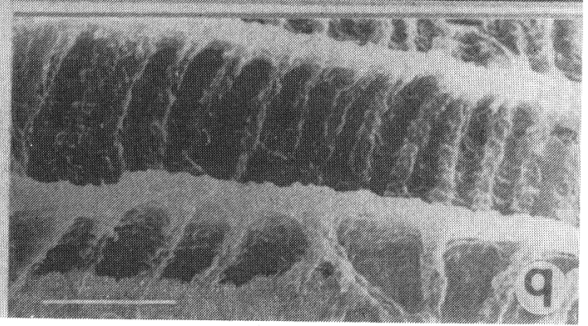


de 10 - $40 \mu \mathrm{m}$, con 1 - 3 capas de células de paredes lignificadas, que se diferencian en dos zonas: la externa está formada por 1 - 2 capas de células cuadradas y pequeñas, mientras que la interna consta de 1 capa compacta sin diferenciación celular (fig. 2 h). De 10 - 25 semillas por cápsula.

Semillas de $1.2-2.0 \times 0.7-1.0 \mathrm{~mm}$, elipsoideas u ovoides. Color grisáceo. Testa crestada, formada por células alargadas tangencialmente, con paredes radiales reticuladas poco o medianamente profundas, de grosor uniforme y pared tangencial externa membranosa colapsada sobre la interna que es reticulada. Entre cada cresta se dispone 1 fila de células, a veces 2, dando una apariencia escalariforme. Epidermis con células isodiamétricas de paredes con refuerzos de lignina excepto la tangencial externa. Hipodermis con 1 - 4 capas de células irregulares de paredes reforzadas. Endotelio con células isodiamétricas, pequeñas de pared tangencial interna gruesa (fig. 2 i-1).

\section{Odontites foliosa Pérez Lara}

Cápsulas de $2.8-4.0 \times 1.5-2.7 \mathrm{~mm}$. De contorno obovado u oblongo, comprimidas, con ápice truncado algo emarginado (Fig. 1 l). Indumento pubescente, adpreso, localizado en la mitad superior, con pelos de $150-400 \mu \mathrm{m}$ (Figs. $1 \mathrm{~m}-\mathrm{q} ; 2 \mathrm{~m}$ ). Pericarpo de $70-90 \mu \mathrm{m}$. Epicarpo con células irregulares de paredes delgadas. Mesocarpo con 2 - 3 capas de células irregulares, colapsadas con frecuencia. Endocarpo de 10 - $30 \mu \mathrm{m}$, con 1 - 2 capas de células cuadradas, pequeñas y lignificadas (Fig. $2 \mathrm{n})$.

Semillas de $1.1-2.0 \times 0.4-0.7$ mm, fusiformes. Color pardo claro. Testa crestada, formada por células alargadas tangencialmente, con paredes radiales reticuladas poco o medianamente profundas, de grosor uniforme y pared tangencial externa membranosa colapsada sobre la interna que es reticulada. Entre cada cresta se dispone 1 fila de células, a veces 2, dando una apariencia escalariforme. Epidermis con células isodiamétricas de paredes con refuerzos de lignina excepto la tangencial externa. Hipodermis con 1 - 3 capas de células irregulares de paredes reforzadas. Endotelio colapsado (Fig. 2 o-q).

\section{Clave de frutos y semillas}

1. Indumento de la cápsula localizado en la zona apical. Testa con 2 filas de células entre cada cresta, presentando un aspecto reticulado .......... O. tenuifolia

1'. Indumento de la cápsula distribuido, al menos, en la mitad superior. Testa con 1 fila de células entre cada cresta, presentando un aspecto escalariforme

2. Cápsula $<4.0 \mathrm{~mm}$ de longitud. Semillas $<0.7$ $\mathrm{mm}$ de anchura, pardo claras O. foliosa

2'. Cápsula $>6.0 \mathrm{~mm}$ de longitud. Semillas $>0.7$ $\mathrm{mm}$ de anchura, grisáceas O. longiflora

\section{DISCUSIÓN}

Las cápsulas de las tres especies examinadas se diferencian fundamentalmente en el tamaño, ya que $O$. foliosa tiene cápsulas bastante más pequeñas que los otros taxones. Por otro lado, el indumento está más extendido y es más denso en $O$. longiflora, mientras que en $O$. foliosa es más laxo y en $O$. tenuifolia está restringido a la zona apical. Sin embargo, en las tres especies las cápsulas son obovoides con ápice truncado, carácter que las relaciona con Nothobartsia y Euphrasia. Las observaciones anatómicas realizadas en los frutos no revelan ninguna diferencia importante entre ellas, aunque se ha visto que $O$. longiflora tiene el pericarpo más grueso y el endocarpo algo más complejo.

En cuanto a las semillas, de acuerdo con Bolliger \& Molau (1992), tienen en el fruto una disposición péndula, son mayores de 0.6 $\mathrm{mm}$ de longitud y la testa es longitudinalmente crestada en los tres taxones. No obstante, en este estudio se ha observado una diferencia en 
las semillas de $O$. tenuifolia, ya que presenta dos filas de células entre cada cresta que le confieren un aspecto reticulado. En $O$. foliosa y $O$. longiflora aparece, por lo general, sólo una fila de células que les da un aspecto escalariforme.

Desde un punto de vista anatómico, las semillas de los tres taxones son bastante semejantes, si bien $O$. longiflora muestra la hipodermis menos degenerada que las otras. La estructura interna observada coincide con la de algunas especies de Rhinanthus y Cordylanthus, miembros también de la tribu Rhinantheae estudiadas por Tiagi (1966) y Chuang \& Heckard (1972) respectivamente. En todas las especies, el endotelio tiene la apariencia de una lámina más o menos gruesa, unida a una capa gruesa de hemicelulosa formada por la pared tangencial externa de las células periféricas del endospermo. Ambas capas forman un revestimiento protector muy eficiente, ya que la testa puede desprenderse con facilidad.

Los caracteres de frutos y semillas estudiados en el presente trabajo no han mostrado diferencias suficientes que respalden claramente el que estas especies se incluyan en géneros distintos. Además, hay que tener en cuenta que algunos caracteres usados por Bolliger (1985) y Bolliger \& Molau (1992) como la presencia de glándulas peltadas 0 cabezas glandulares con divisiones horizontales son muy variables en Odontites s.s. pudiendo incluso estar presentes o ausentes. Esto parece indicar que este género es muy polimorfo y que las diferencias entre los caracteres que han permitido separarlo en varios géneros, entrarían en el rango de variabilidad de Odontites s.l. Por ésto, las especies examinadas se han incluido en el género Odontites como hicieron anteriormente Webb \& Camarasa (1972) y Valdés (1987).

AGRADECIMIENTOS. Los autores desean expresar su agradecimiento al Dr. P. J. López-
González por la realización de los dibujos y al Servicio de Microscopía Electrónica de la Universidad de Sevilla la ayuda prestada, en especial a Dña. A. Fernández por la preparación de las muestras en punto crítico.

\section{BIBLIOGRAFÍA}

BENTHAN, G. - 1846- Ordo CXLII. Scrophulariaceae. In A. P. DE CANDOLLE (ed.). Prodromus Systematis naturalis Regni Vegetabilis, 10. Paris.

BOLLIGER, M. -1985-Die Drüsenhaare der Gattung Odontites Ludwig (Scrophulariaceae) und ihre systematische Bedeutung. Bot. Jahrb. Syst., 107: 153-175.

BOLLIGER, M. \& L. WICK -1990- The pollen morphology of Odontites (Scrophulariaceae) and its taxonomic significance. Pl. Syst. Evol., 173: $159-178$.

BOLLIGER, M. \& U. MOLAU -1992- Nothobartsia, a new genus of Scrophulariaceae from southwest Europe. Pl. Syst. Evol., 17: 59-71.

CHUANG, T. I. \& L. R. HECKARD -1972- Seed coat morphology in Cordylanthus (Scrophulariaceae) and its taxonomic significance. Amer. J. Bot., 59: 258-265.

FONT QUER, P. -1977-Diccionario de Botánica. Barcelona.

JOHANSEN, D. A. -1940- Plant microtechnique. New York.

LINNEO, C. -1753- Species Plantarum. Holmiae.

ROTHMALER, W. -1943- Zur Gliederung der Antirrhineae. Feddes Repert., 52: 16-39.

STEARN, W. T. -1992-Botanical Latin. London.

TIAGI, B. -1966-Development of the seed and fruit in Rhinanthus major and R. serotinus. Amer. $J$. Bot., 53(7): 645-651.

VALDÉS, B. -1987-Scrophulariaceae. In B. VALDÉS et al. (eds.) Flora de Andalucía Occidental, 2. Barcelona.

WEBB, D. A. \& J. M. CAMARASA -1972Odontites. In T. G. TUTIN et al. (eds.) Flora Europaea, 3. Cambridge.

Aceptado para su publicación en Junio de 1996

Dirección de los autores. Departamento de Biología Vegetal y Ecología. Universidad de Sevilla. Apartado 1095. 41080 Sevilla. 